\title{
The promise of quantitative phase imaging and machine learning in medical diagnostics
}

\author{
Yaw Ansong JNR ${ }^{1^{*}}$ \\ ${ }^{1}$ Biomedical Engineering Department, University of New Haven, 300 Boston Post Road, West Haven CT, USA
}

\begin{abstract}
Quantitative phase imaging (QPI) is a method of phase-contrast microscopy which quantifies the phase shift that occurs when light passes through an optically dense object. Machine learning relies on patterns and inference to study algorithms and statistical models with the goal of performing tasks without explicit instructions. QPI provides an enormous amount of information about cells. In the past, however, applying information obtained from QPI based cell profiling into practical translational solutions has been challenging due to limited access to analytical tools capable of making full sense of this data. Recent advances in artificial intelligence and machine learning, however, suggest opportunities in applying QPI to medical diagnostics. This paper discusses how artificial intelligence, machine learning, and quantitative phase imaging can be used in medical diagnostics.
\end{abstract}

Keywords: artificial intelligence; QPI; quantitative phase imaging; computational; deep learning; machine learning

\begin{abstract}
*Corresponding author: Yaw Ansong JNR, Biomedical Engineering Department, University of New Haven, 300 Boston Post Road, West Haven CT, United States of America. Tel. +1-203343-8629; Email: yanso2@unh.newhaven.edu
\end{abstract}

Received 29 May 2019; Accepted 14 June 2019; Published 22 June 2019

Citation: Ansong JNRY. The promise of quantitative phase imaging and machine learning in medical diagnostics. J Med Sci Res. 2019; 7(3):63-65. DOI: http://dx.doi.org/10.17727/JMSR.2019/7-e1

Copyright: (C) 2019 Ansong JNRY et al. Published by KIMS Foundation and Research Center. This is an open-access article distributed under the terms of the Creative Commons Attribution License, which permits unrestricted use, distribution, and reproduction in any medium, provided the original author and source are credited.

\section{Introduction}

Quantitative phase imaging (QPI) is a method of phase-contrast microscopy which quantifies the phase shift that occurs when light passes through an optically dense object [1,2]. Unlike other conventional phase-contrast methods, QPI creates a phase shift image independent of the bright field image. The method is very useful in providing quantitative information about cells such as the morphology and cell dry mass [3]. This method can, therefore, provide enormous information about cells. In the past, however, applying information obtained from QPI based cell profiling into practical translational solutions has been challenging due to limited access to analytical tools capable of making full sense of this data [4]. Recent advances in Artificial intelligence and machine learning [5] suggest opportunities in applying QPI to medical diagnostics [6]. The goal 
of this paper is to discuss artificial intelligence, machine learning, QPI and how these techniques can be combined in medical diagnostics.

\section{Artificial intelligence}

Artificial intelligence (AI) can be described as machines (or computers) that mimic "cognitive" functions usually associated with the human mind, for example; "learning" and "problem-solving" [7]. A more comprehensive definition of AI is "a system's ability to correctly interpret external data, to learn from such data, and to use those learnings to achieve specific goals and tasks through flexible adaptation" [8]. It is very common for tasks previously thought to be unachievable by machines to be removed from the definition of AI once machines achieve that task. This is known as the AI effect [9]. AI involve the use of algorithms.

\section{Machine learning}

Machine learning, seen as a sub-set of artificial intelligence, [10] relies on patterns and inference to study algorithms and statistical models with the goal of performing tasks without explicit instructions [11]. In machine learning, the algorithm basically learns the associations of predictive data using examples in data [10]. Using exploratory data analysis through unsupervised learning is known as data mining [12].

\section{Deep learning}

Deep learning, a machine learning method based on artificial neural networks, involves feeding an algorithm with large data sets with the goal of discovering the representations needed for classification or detection [13]. It may be supervised or unsupervised.

\section{Supervised learning}

Supervised learning, is the machine learning task of mapping input to an output based on input-output pairs [14]. The outputs of interest in supervised learning are typically defined by a human supervisor. The goal of learning associations is to be able to predict future outputs based on input after the algorithm has been trained to make predictions.

\section{Unsupervised learning}

Unsupervised learning, unlike supervised learning, learns associations in a dataset without any external definition of associations of interest. It, therefore, identifies previously undiscovered associations instead of relying on known ones [10].

\section{Quantitative phase imaging and deep learning in cell diagnostics}

The diverse properties of cells in the body changes based on pathophysiological conditions [15]. It is a known fact for example that various parasitic infections and diseases alter red blood cell properties [16]. Alterations in the deformability of red blood cells have for example been documented in cases of malaria, sickle cell anemia, diabetes, just to name a few [17]. Complete blood count, the universally accepted method for hematological examination of red cell properties, is population-based and fails to focus on individual red cell properties [4]. Profiling individual red cells can, therefore, be a game-changer in screening diseases based on individual red blood cells [18]. QPI, a method that uses the differences in intracellular refractive index distribution as an imaging contrast for label-free imaging, [19] can serve as a viable method for profiling individual cells. The challenge, however, is that the data will be accompanied by variations in each cell, a challenge which hitherto would mean difficulty in analyzing the resultant data due to lack of proper analytics tools. With the improvement in computing power and deep learning however, we can increasingly make sense of this massive data. For example, QPI and machine learning have been combined to help in the diagnosis of malaria infections [20], sickle cell, [21] and sperm cell analysis [22]. It is therefore proposed that, in the future, QPI can be combined with machine learning in order to provide novel diagnostic solutions.

\section{Ethical considerations}

It is obvious that large amounts of data sets will be needed in order to these train machine learning algorithms. It is essential that biases in the patient selection are avoided in order to ensure that samples used are representative enough. It is also important to ensure that data used in AI research are ethically sourced and not weaponized to attack vulnerable populations.

\section{Final thoughts}

There is great promise in combining quantitative phase imaging with machine learning and it can 
potentially lead to affordable, groundbreaking diagnostic solutions.

\section{Conflicts of interest}

Author declares no conflict of interest.

\section{References}

[1] Rubin SE. Plant Microtechnique and Microscopy. Oxford University Press. 1999.

[2] Cuche E, Bevilacqua F, Depeursinge C. Digital holography for quantitative phase-contrast imaging. Opt Lett. 1999; 24(5):291-293.

[3] Zangle T, Teitell M. Live-cell mass profiling: an emerging approach in quantitative biophysics. Nat Methods. 2014; 11(12):1221-1228.

[4] Kim G, Jo Y, Cho H, Min HS, Park Y. Learning-based screening of hematologic disorders using quantitative phase imaging of individual red blood cells. Biosens Bioelectron. 2019; 123:69-76.

[5] Bishop CM. Pattern Recognition and Machine Learning. Springer. 2006.

[6] Cruz JA, Wishart DS. Applications of machine learning in cancer prediction and prognosis. Cancer Inform. 2007; 2:59-77.

[7] Russell Stuart J, Norvig Peter. Artificial Intelligence: A Modern Approach. 3rd Edition. Upper Saddle River, New Jersey: Prentice Hall. 2009.

[8] Andreas K, Michael H. Siri, Siri, in my hand: Who's the fairest in the land? On the interpretations, illustrations, and implications of artificial intelligence. Business Horizons. 2009; 62(1):15-25.

[9] McCorduck, Pamela. Machines Who Think. 2nd edition. Natick, MA: A. K. Peters, Ltd. 2004.

[10] Panch T, Szolovits P, Atun R. Artificial intelligence, machine learning and health systems. J Glob Health. 2018; 8(2):020303.

[11] John RK, Forrest HB, David A, Martin AK. Automated Design of Both the Topology and Sizing of Analog Electrical Circuits Using Genetic. 1996.

[12] Friedman, Jerome H. Data Mining and Statistics: What's the connection?. Computing Science and Statistics. 2009; 29(1):3-9.

[13] LeCun Y, Bengio Y, Hinton G. Deep learning. Nature. 2015; 521(7553):436-444.

[14] Russell SJ, Norvig P. Artificial Intelligence: A Modern Approach. Third Edition, Prentice Hall. 2010.

[15] Diez-Silva M, Dao M, Han J, Lim CT, Suresh S. Shape and Biomechanical Characteristics of Human Red Blood Cells in Health and Disease. MRS Bull. 2010; 35(5):382-388.

[16] Chandramohanadas R, Park Y, Lui L, Li A, Quinn D, et al. Biophysics of malarial parasite exit from infected erythrocytes. PLoS One. 2011; 6(6):e20869.

[17] Tomaiuolo G. Biomechanical properties of red blood cells in health and disease towards microfluidics. Biomicrofluidics. 2014; 8(5):051501.

[18] Weatherall DJ. Systems Biology and Red Cells. New Engl J Med. 2011; 364(4): 376-377.

[19] Wolf E. Three-dimensional structure determination of semi-transparent objects from holographic data. Optics Communications. 1969; 1(4):153-156.
[20] Go T, Kim JH, Byeon H, Lee SJ. Machine learning-based in-line holographic sensing of unstained malaria-infected red blood cells. J Biophotonics. 2018 Sep;11(9):e201800101.

[21] Javidi B, Markman A, Rawat S, O'Connor T, Anand A, et al. Sickle cell disease diagnosis based on spatio-temporal cell dynamics analysis using 3D printed shearing digital holographic microscopy. Opt Express. 2018; 26(10):1361413627.

[22] Mirsky SK, Barnea I, Levi M, Greenspan H, Shaked NT. Automated analysis of individual sperm cells using stain-free interferometric phase microscopy and machine learning. Cytometry A. 2017; 91(9):893-900. 